\section{Granulozytopoese, neutrophile}

H. Baum

Institut für Laboratoriumsmedizin, Mikrobiologie und Blutdepot, Regionale Kliniken Holding RKH GmbH, Ludwigsburg, Deutschland

Englischer Begriff neutrophilic granulocytopoiesis

Definition Ausdifferenzierung und Reifung der myeloischen Vorläuferzellen unter dem Einfluss von Wachstumsfaktoren im Knochenmark zum neutrophilen Granulozyten.

Beschreibung Die neutrophile Granulozytopoese beschreibt die Reifung von der myeloischen Vorläuferzelle (GEMMCFU) zur reifen Effektorzelle, dem segmentierten neutrophilen Granulozyten. Die früheste, morphologisch nachweisbare Zelle der neutrophilen Granulopoese ist der Myeloblast
( $\triangleright$ Myeloblasten), der aus der myeloischen Vorläuferzelle nach Induktion mit spezifischen Wachstumsfaktoren ( $\triangleright$ GCSF, \ GM-CSF, IL-3) entsteht. Nach Teilung und Ausdifferenzierung können unterschieden werden: $>$ Promyelozyt, - Myelozyten, - Metamyelozyten, stabkerniger Granulozyt $(\triangleright$ Granulozyten, stabkernige) und segmentkerniger neutrophiler Granulozyt. Bis zum Reifungsstadium des Metamyelozyten besitzen die Zellen noch die Fähigkeit zur Teilung. Im Reifungsstadium des Promyelozyten können dann erstmals Granula, die Primärgranula, nachgewiesen werden, die im Laufe der Ausreifung durch die spezifischen neutrophilen Granula der reifen Formen ersetzt werden.

\section{Literatur}

Nerl C (1993) Zellen der Granulopoese. In: Begemann H, Rastetter J (Hrsg) Klinische Hämatologie, 4. Aufl. Georg Thieme Verlag, Stuttgart, S 66-78 